\title{
Satellite Backhauling for Next Generation Cellular Networks: Challenges and Opportunities
}

\author{
Yekta Turk ${ }^{\diamond}$, Engin Zeydan* \\ ${ }^{\diamond}$ Ericsson Research, Istanbul, Turkey, 34396. E-mail: yekta.turk@ericsson.com \\ *CTTC, Castelldefels, Barcelona, Spain, 08860. Email: engin.zeydan@cttc.cat
}

\begin{abstract}
Recently, studies on integration of satellitebased communication with terrestrial next-generation cellular networks (5G) have emerged in academia, standardization bodies and industry due to extended coverage and service enrichment possibilities for Mobile Network Operators (MNOs). In this article, we (i) first review the existing studies on satellite-based mobile network architectures and standardization efforts, (ii) propose a new satellite-based backhaul architecture for mobile communications and evaluate comparative end-to-end performance of both proposed satellite based backhaul and pure terrestrial architecture deployment scenarios through realistic experimental evaluations in terms of various Key Performance Indicators (KPIs) used by MNOs (iii) discuss about encountered limitations and challenges well as the open issues of the experimented satellite-based mobile backhaul architecture (iv) and finally provide a reference architecture and some guidelines towards designing a satellite based backhaul for next generation cellular networks from the perspective of MNOs.
\end{abstract}

Index Terms-satellite, cellular networks, experimental, architecture, operators.

\section{INTRODUCTION}

Satellite networks are going to be a complementary component of next generation cellular communication networks [1]. This trend has taken place under the circumstances of combining high coverage capabilities of satellite networks and large number of connection capabilities of cellular networks by varieties of novel features, i.e. Internet of Things, edge and cloud computing, big data, etc. Together with integrated satellite and terrestrial networks, benefits such as providing an efficient way of long distance connectivity to large regions based on the requirements, scalability, flexibility and

${ }^{*}$ Corresponding author.

This work was partially funded by Spanish MINECO grant TEC2017-88373-R (5G-REFINE), by Generalitat de Catalunya grant 2017 SGR 1195 and by Turkey Scientific and Technological Research Council 1515 Program, Project 5169902. advanced broadcasting services, freeing Mobile Network Operators (MNOs) from investment costs and configuration burdens of fiber, enabling reliability from man-made or natural events based outages can be envisioned. Moreover as a backhaul alternative, Line-of-Sight (LOS) satellite links can be used to relay mobile traffic in access network directly into core network $(\mathrm{CN})$ without requiring several hops.

These benefits of using satellite communication affect not only rural and remote areas, but also urban areas where satellites can provide an alternative backhaul solution for bandwidth extension purposes during high traffic demand. Today, emerging techniques for integrated satellite and terrestrial networks are at exploratory phase including many security, regulatory and technical related challenges that still remain to be addressed before realizing the envisioned potentials. For enhanced integration of satellite with $5 \mathrm{G}$ standards, Satellite Network Operators (SNOs) have also started to support 3GPP activities [1]. In this article, in addition to experimenting a real-world integrated satellite-terrestrial network that exploits potential benefits of satellitebased mobile backhaul, we also focus on limitations and challenges of the experimental trial and provide a reference architectural deployment scenario for next generation integrated satellite and terrestrial networks.

\section{OVERVIEW OF ARCHITECTURES}

Different architectural studies to enable possible integration of satellite communication into terrestrial cellular infrastructure are under study as activities within Standard Developing Organizations (SDOs) and relevant research and industrial projects [1]-[4]. Current standardization efforts on 3rd Generation Partnership Project (3GPP) are mostly concentrating on using New Radio (NR)-Uu over satel- 


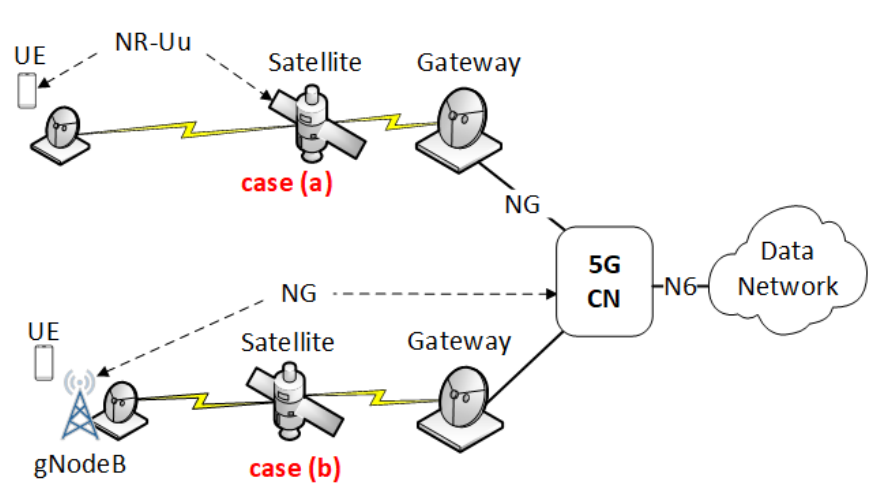

Figure 1: Satellite communication over access network (NR-Uu interface) in case (a) and over backhaul network (NG interface) in case (b).

lite connections to gNodeB. 3GPP has recently initiated a Release-16 technical requirement document that investigates solutions to support NR for NonTerrestrial Networks (NTNs) [1] whereas satellite based backhaul communication is on the horizon. Seamless integration of satellite components with terrestrial networks is studied with H2020 SANSA project [2]. Similarly, H2020 project Sat5G investigates solutions to enable ubiquitous $5 \mathrm{G}$ coverage thanks to cost effective "plug and play" integration of satellite infrastructure of SNOs with backhaul connection of terrestrial 3GPP based infrastructure of MNOs [3]. SATis5 project highlights benefits of satellite technology for main 5G use cases [4].

Fig. 1 presents two different satellite based mobile architectures. The satellite's role is mainly divided into two functionalities. In case (a), satellite is envisioned to provide NR-Uu interface for user equipments (UEs) and in case (b) it acts as backhaul satellite which is on NG interface in 5G. These access architectures have their own trade-offs when integration with mobile network infrastructures are considered. For example, in case (a), caching on base stations (BSs) [5] is not an easy option for MNOs due to availability of third party vendors and the challenges of case (b) are detailed in Section IV.

\section{PRACTICAL EXPERIMENTAL VALIDATION}

In this section, we present results of our experimental test setup that utilizes satellite-based backhaul architecture for $4 \mathrm{G}$.

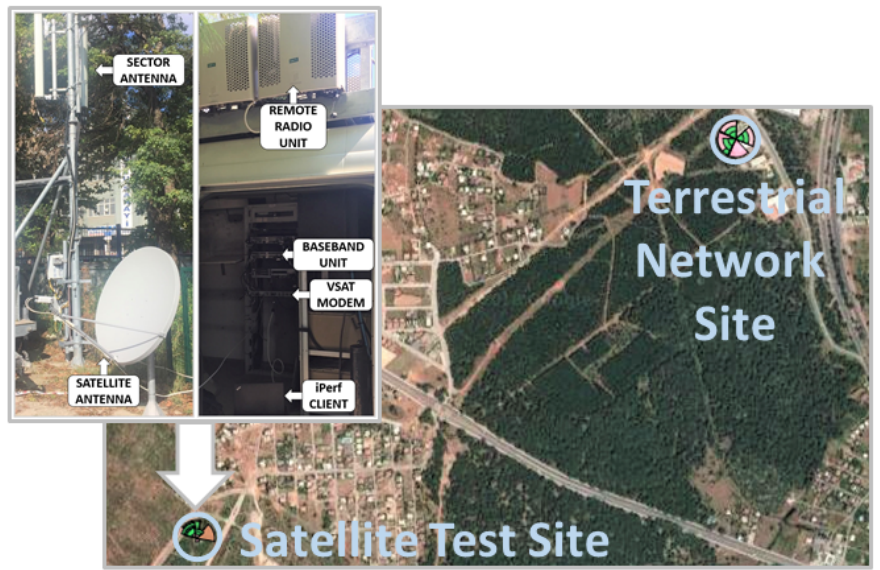

Figure 2: Experimental test setup and locations of both satellite and terrestrial network sites.

\section{A. Satellite-Based Architecture over S1}

Our experimental proposal studies a new architecture where S1 interface is used over satellite link transmission. This architecture has also certain advantages compared to NR-Uu based satellite connectivity. Fig. 2 shows locations of satellite and terrestrial test-sites and pictures of different types of network devices that are used during our experimental trials. These components are: satellite antenna, sector antenna, Baseband Unit (BBU), Remote Radio Unit (RRU), Very Small Aperture Terminal (VSAT) Modem and iPerf Client. Details of the test-bed can be found in [6].

Case (a) S1 path of Fig. 3 represents a regular end-to-end system architecture of the utilized testbed in terrestrial network site whereas case (b) $\mathrm{S} 1$ path of Fig. 3 is the proposed integrated satellite and cellular network architecture. In case (b) S1 path of Fig. 3, S1 interface between eNodeb and Evolved Packet Core (EPC) is carried via geosyn-

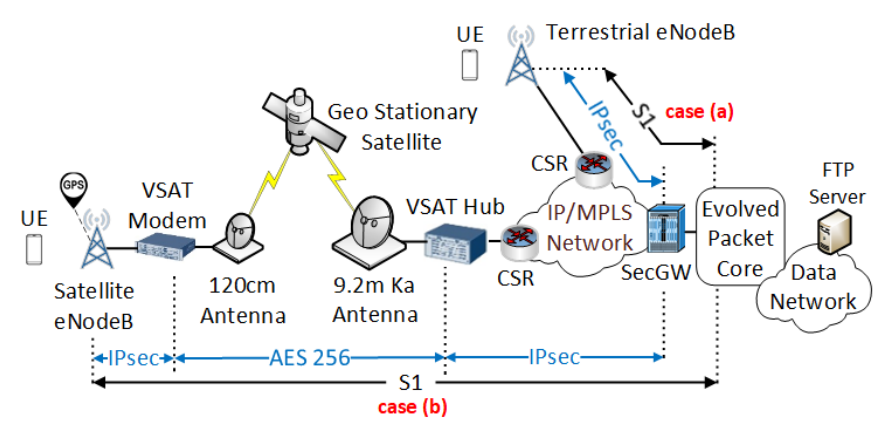

Figure 3: Test architectures for terrestrial network site (case (a)) and satellite test site (case (b)). 
chronous (GEO) satellite. There is an Ethernet connection between satellite eNodeB and VSAT modem through Internet Protocol Security (IPsec) tunnel for end-to-end encryption. Both VSAT modem VSAT hub are capable of setting up IPsec tunnel and user plane (UP) acceleration. Note that between VSAT modem and hub, Advanced Encryption Standard (AES)-256 is utilized. The ground station has $9.2 \mathrm{~m}$ $\mathrm{Ka}$ antennas and a VSAT hub. VSAT hub establishes a new IPsec tunnel with the Security Gateway (SecGW). However, since satellite eNodeB's S1 traffic is transmitted via satellite link in this topology, mobile Cell Site Router (CSR) is installed at the ground station where VSAT hub is located so that connectivity over fixed network operator's infrastructure via a leased line can be established. SecGW is used to terminate IPsec tunnels so that $\mathrm{S} 1$ traffic can reach to the EPC as given in case (b) S1 path of Fig. 3. It is important to reduce the end-to-end delay to ensure that quality-of-service (QoS) requirements of end-users are met. For this reason, GPRS Tunneling Protocol (GTP)-U acceleration (GTP-A in short) is applied at both VSAT modem and hub [7], [8] to provide low end-to-end delay.

\section{B. Experimental Performance Analysis}

Fig. 4 shows the violin plot comparisons of normalized Downlink (DL) Key Performance Indicator (KPI) values illustrating both quartiles and density plots to show the distribution shapes for both terrestrial and satellite eNodeBs. During our experiments, we observed higher link quality metrics (both Channel Quality Indicator (CQI) (median around 100\%) and Modulation Coding Scheme (MCS) index (median around 100\%)) in satellite eNodeB compared to terrestrial eNodeB (CQI (median around $23.88 \%$ ) and MCS index (median around $58.37 \%$ ). In satellite eNodeB, the standard deviation of resource block (RB) utilization is observed to be larger (around 16.88) compared to terrestrial eNodeB (around 7.37). There is high standard deviation on RB utilization ratio for satellite eNodeB as a consequence of incomplete transmit buffer. This incompleteness is due to irregular Protocol Data Unit (PDU) receptions caused by delays and jitter in satellite links. Satellite eNodeB cannot schedule PDUs appropriately at Medium Access Control (MAC) layer owing to incomplete transmit buffer.

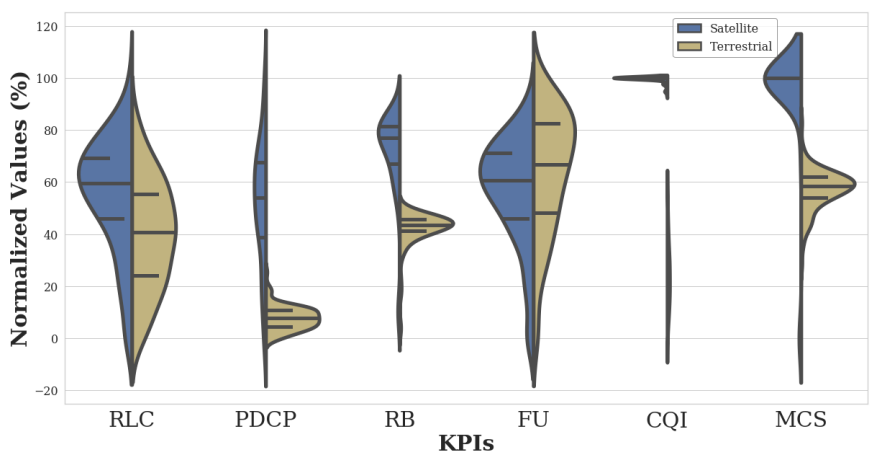

Figure 4: Violin plots for comparisons of normalized KPI values in both terrestrial and satellite eNodeBs.

Fig. 4 clearly validates the lower Frame Usage (FU) ratio (with median of $60.66 \%$ ) in satellite eNodeB compared to the terrestrial eNodeB (with median of $66.87 \%$ ). This is mainly due to high number of UEs in terrestrial eNodeB. The standard deviation on FU ratio is narrower on satellite $\mathrm{eN}$ odeB (around 20.83) compared to terrestrial eNodeB (around 23.97). This is mainly due to poor radio conditions in terrestrial eNodeB. Similarly, Fig. 4's Radio Link Controller (RLC) and Packet Data Convergence Control (PDCP) median normalized values indicate that satellite eNodeB has high values (59.68\% and $53.97 \%$ respectively) compared to terrestrial eNodeB (40.67\% and $7.76 \%$ respectively).

\section{Effects of Using Satellites for Mobile Backhaul}

In this subsection, we describe three main outcomes of the practical experimental results as follows.

Unbalanced RB utilization and FU ratio distributions: The intrinsic delay and jitter of satellite link cause the standard deviation of RBs utilization for satellite eNodeB to be higher than terrestrial eNodeB. Note that there is only one test UE that is connected to the satellite eNodeB with good channel conditions. Although mass of the RB utilization and FU ratio distributions are expected to concentrate on high values, distributions with high standard deviations have been monitored. This is because the transmit buffer in the satellite eNodeB is irregularly occupied by PDCP PDUs. Consequently, the satellite eNodeB was unable to schedule and transmit PDCP PDUs properly because the transmit buffer size was not complete during the scheduling interval. Hence, the distributions of RB utilization and FU ratio are more scattered than expected. 
RLC and PDCP PDU issues: Although channel quality is good for satellite eNodeB, high number of PDUs are generated at PDCP layer in comparison with RLC layer. Note that during regular scheduling operation in terrestrial eNodeB, RLC layer is in charge of segmenting all received PDCP PDUs for transmission. On the other hand in satellite eNodeB, PDCP PDUs are aggregated in RLC layer. Therefore, a transmit buffer problem is expected in satellite eNodeB when many UEs are connected and produce huge number of PDCP PDUs.

Trade-off between the caching size and the supported number of UEs: GTP-A method exploits caching to accelerate traffic in VSAT modem and hub. From experimental results, a relationship between caching size, acceleration rate of GTP-A and supported number of UEs can be deducted. If caching size is large, the acceleration rate will be increased by GTP-A method. This will decrease the latency in the satellite link, but will also produce huge number of PDCP PDUs which will eventually reduce the supported number of UEs for satellite eNodeB. Therefore, when designing a satellite based mobile backhaul system, trade-off analysis between acceleration rate and support for high number of UEs are required.

\section{LiMITATIONS AND CHALLENGES OF EXPERIMENTAL TRIALS}

\section{A. User Plane Traffic Acceleration}

Propagation delays and Doppler shifts can be 300 times and 126 times larger than at terrestrial links, respectively [3]. Due to long propagation delays that the signal experiences, acceleration is required for Stream Control Transmission Protocol (SCTP) (in control plane (CP)) and especially for GTP-U (in UP) messages. eNodeB's CP is not highly sensitive to the delay in the network as UP. However, traffic acceleration is required for GTP-U messages, so that the UP can operate successfully. To achieve this, UP messages that are encapsulated within IPsec tunnel need to be decrypted first. Relevant patents in [7] and [8] propose UP acceleration techniques. For both Uplink (UL) and DL, GTP-A procedure decapsulates GTP-U messages, caches the payload and applies a web acceleration method to this payload at one side (either VSAT hub or modem) and re-encapsulates them at the other side (either
VSAT hub or modem) as originally sent GTPU messages. During this process, tunnel endpoint identifier (TEID) fields of GTP-U messages that are used in one side are sent to the other side for original re-encapsulation of GTP-U messages. Hence, these TEID fields are no longer repeated for each messages. In experimental tests, disabling traffic acceleration led to disconnection of data traffic even if the CP link was up and running. GTP-A procedure enabled improvements in Internet Protocol (IP) traffic performance by mitigating the effects of the delay in satellite link.

\section{B. Fragmentation of IPsec Tunnel}

MNOs mostly use IPsec to prevent security risks for data traffic flowing between eNodeB and EPC [9]. However, considering the satellite transmission, traffic acceleration is required for effective data transmission over GTP tunnel that is setup between eNodeB and Serving Gateway (S-GW). Unfortunately, encrypted IPsec traffic prevents traffic acceleration. In this case, VSAT modem must decode the encryption, accelerate the data traffic, and then re-encrypt it. Since encrypted data cannot be accelerated to apply proper GTP-A process, IPsec tunnel needs to be terminated at VSAT modem for traffic between eNodeB and VSAT modem and at VSAT hub for traffic between SecGW and VSAT hub. Due to inefficiency of IPsec usage via satellite [10], AES-256 is used for the traffic over the satellite link between VSAT modem and hub. Finally, a secure communication between eNodeB and EPC is established by setting up two IPsec tunnels and one AES-256 encryption. In case (a) S1 path of Fig. 3, blue arrows indicate termination points for IPsec tunnel in a regular terrestrial network. In case (b) S1 path of Fig. 3, IPsec and AES-256 connection end-points and fragmentation location are shown in three separate blue arrow lines.

\section{Control Plane Link Setup}

At eNodeB, there are retransmission and acknowledgement timers that need to be adjusted by MNOs to obtain reliable $\mathrm{CP}$ links. Re-transmission timers are set to wait for predefined time interval and resend $\mathrm{CP}$ messages that are not delivered during this interval. The acknowledgement timer sets the waiting period for the acknowledgement of sent messages. Different $\mathrm{CP}$ timer values can 


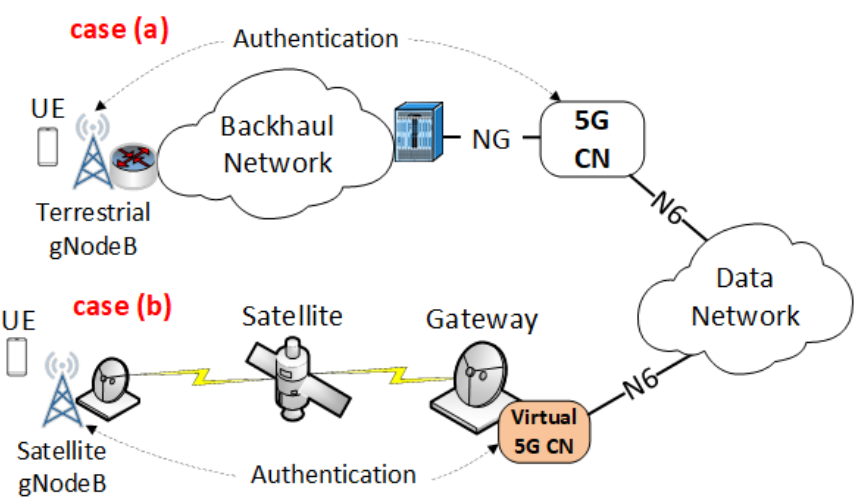

Figure 5: Authentications of terrestrial gNodeB in case (a) and satellite gNodeB in case (b).

be set for eNodeBs based on the requirements of MNO. During our experimental trials, we detected that adjusting those timer values with same values as the ones used in terrestrial network settings may cause the CP link to be continuously flapped. In this case, due to inherent latency introduced by satellite transmission, eNodeB can not receive messages within pre-defined time intervals. Therefore, manual adjustments of these two timers need to be done.

\section{Synchronization}

Synchronization messages are transmitted to eNodeBs from clock sources that are deployed by MNO at various suitable locations of the network. During our experiments, accurate synchronization messages could not be delivered to the satellite test eNodeB due to inherent transmission delay. For this reason, a global positioning system (GPS) antenna is installed and connected to the satellite test eNodeB, so that correct synchronization messages can be provided over GPS.

\section{Open Issues and Possible Solutions WITH SATELLITE BACKHAULING}

\section{A. Security Concerns}

Since IPsec in 3GPP security architecture has problems with satellite transmission, Datagram Transport Layer Security (DTLS) can used as an alternative 3GPP security profile [9]. DTLS is User Datagram Protocol (UDP) based, hence UP PDUs can be encrypted and decrypted either at $5 \mathrm{G} \mathrm{CN}$ or SecGW. The positioning of UP Acceleration as one of the features embedded both into gNodeB and $5 \mathrm{G}$
$\mathrm{CN}$ will eliminate the IPsec fragmentation problem. Therefore, DTLS will ensure end-to-end security.

gNodeB authentication: All gNodeBs in the infrastructure of MNOs need to be authenticated. This is a requirement for both terrestrial gNodeBs (which are traditionally authenticated at $5 \mathrm{G} \mathrm{CN}$ ) as given in case (a) of Fig. 5 and satellite gNodeBs. Authentication for satellite gNodeBs will take longer time due to satellite transmission delay and possible transmission quality problems between the satellite gateway and the remotely located $5 \mathrm{G} \mathrm{CN}$. This could impact the experience of users connected to the satellite gNodeBs. Due to the introduced delay, the $\mathrm{CP}$ parameters of the $5 \mathrm{G} \mathrm{CN}$ where satellite gNodeBs are authenticated are different from the $5 \mathrm{G} \mathrm{CN}$ that terrestrial gNodeBs are authenticated. Moreover, transmitting the satellite gNodeB's traffic to a remote $5 \mathrm{G} \mathrm{CN}$ creates extra overload, cost as well as unnecessary connections. As a result, authentication procedure of satellite gNodeBs need to be separated from terrestrial gNodeBs inevitably. An applicable solution is to authenticate satellite gNodeBs via a virtual $5 \mathrm{G} \mathrm{CN}$ that is positioned inside satellite gateway locations (which are usually in disaster free geographic zones of the country) as given in case (b) of Fig. 5. Positioning a virtual $5 \mathrm{G} \mathrm{CN}$ inside satellite gateway locations has two main benefits. First, it provides a more robust network infrastructure against natural disasters such as hurricanes, earthquakes, etc. Second, low latency communication within the backhaul network can be ensured.

\section{B. Network Governance}

Compared to terrestrial networks, the ownership of satellite equipment (VSAT modem and hub) is obligatory to be with MNOs in some countries (e.g. in Turkey) and the ownership is restricted to be with 3rd parties (such as satellite technology providers) to provide mobile communication over satellite. Since this will mean extra investments on MNOs for these equipment, in the scenario when mobile traffic is carried over satellite transmission with the equipment owned by 3rd party satellite companies, local government agencies will demand to have customized mobile communication license for satellite providers. On the other hand, the transmission cost per bit in satellite links is higher than traditional terrestrial transmission used in cellular 
networks. For example, per $\mathrm{Mb}$ consumption of satellite link can cost up to $\$ 350$ [11] (e.g. in comparison, a BS with $20 \mathrm{Mhz}$ bandwidth can reach up to $150 \mathrm{Mbps}$ speed). To avoid this cost, methods such as Radio Access Network (RAN) and transport network sharing can be utilized, where the gNodeB investment and the operating expenditure of the satellite link can be shared among multiple MNOs.

\section{In-network Processing Capabilities}

Most MNOs utilize Self-Organizing Network (SON) systems that tune network related features and parameters to optimize RAN and transport network. In case, RAN and transport network equipment need to be integrated with satellite systems, new optimization algorithms and expertise will need to be introduced into already existing SON architecture of MNOs. On the other hand, this is not a direct requirement for terrestrial networks.

The ability to communicate over satellite can be embedded into gNodeB rather than to a separate VSAT modem which need to be managed separately. This embedding functionality brings some advantages. One example advantage is the possibility to utilize different KPI in gNodeB to adjust $\mathrm{CP}$ parameters dynamically. Another advantage is to integrate dynamic IP payload (de)compression functionality at gNodeB based on service requirements. This embedding is an additional functionality which is not needed for terrestrial network cases.

\section{Signaling and Timing Constraints}

In terrestrial network due to low latency in backhaul networks, existing $\mathrm{CP}$ signalling methods are sufficient to provide robust, reliable and uninterrupted communications. However in satellite based backhaul, CP signalling needs enhancements to achieve the equivalent performance with terrestrial networks. Note that IMT-2020 requirement on CP signaling is more stringent in 5G than LTE [12]. Therefore, methods such as SCTP with Potentially Failed (SCTP-PF) can be used as a solution against delay sensitivity [13].

Synchronization messages cannot be carried over the satellite between clock sources and gNodeB due to satellite transmission delay in satellite based backhaul. This problem can be solved by using the appropriate profile of the synchronization protocol specified by ITU [14]. This profile must also be supported by both satellite and gNodeB, so that delay request messages can be tolerable up to and not exceeding two seconds in the network. Specifying an offset can be another solution to synchronization problem if the satellite delay is determined dynamically by a test signal sent by gNodeB. In this case, proper synchronization can be achieved by dynamically subtracting this measured offset value from the detected synchronization time by gNodeB.

\section{DESIGN RECOMMENDATION}

This section discusses a new reference satellite based backhaul architecture design that is suitable for next generation $5 \mathrm{G}$ networks. We aim to identify the required additional functionalities both at terrestrial and satellite network levels to mitigate the shortcomings observed in experimental results and improve 3GPP proposed architectures in Release 16.

Fig. 6 illustrates the reference satellite backhaul architecture for 5G. In this evolutionary architecture, the aim is to develop "plug \& play" architecture for telecommunication operators and vendors. Therefore, independent improvements and feature updates in satellite industry and terrestrial network infrastructure can be integrated together. The only exception is the need for newly defined ITU-T profile support for SNOs which supports time offsetting adjustments due to delay [14]. The endto-end connectivity is secured with DTLS protocol which solves the fragmented IPsec connection in case (b) S1 path of Fig. 3. Moreover, gNodeB and $5 \mathrm{G} \mathrm{CN}$ are capable of necessary functionalities such as $\mathrm{CP}$ adaptive configuration, $\mathrm{CP}$ quick failover, $\mathrm{CP}$ and UP-acceleration, scheduling and caching coordination, payload (de)compression and gNodeB authentication. Hence, terrestrial infrastructure can interoperate with SNOs infrastructure. In reference architecture, VSAT terminal allows connection over satellite networks acting as a modem to convert satellite signals into Ethernet.

For optimal operation of the $\mathrm{CP}$ signaling, timer values must be updated dynamically depending on observed satellite delays. This is achieved by $\mathrm{CP}$ adaptive configuration feature. These values need to be adjusted on both $\mathrm{RAN}$ and $5 \mathrm{G} \mathrm{CN}$ in an adaptive manner. $\mathrm{CP}$ quick failover should be identified quickly and recovered when the $\mathrm{CP}$ signaling is lost as suggested in [13]. UP and CP-acceleration features allow acceleration similar to GTP PDUs for 


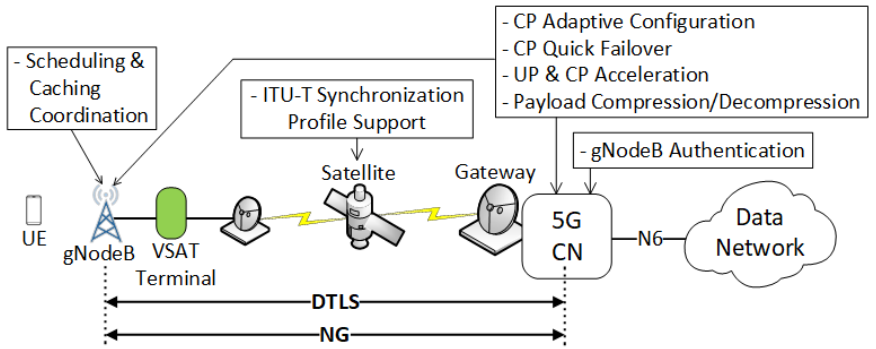

Figure 6: A reference architecture for satellite-based backhaul in next generation cellular networks.

UP as utilized in our experimental results. For CPacceleration, similar mechanisms used in [15] can be utilized. Payload (de)compression feature enables in-network data (de)compression to compensate for the experienced congestion due to inherent delay in the network. Lastly, based on our experimental observation trade-offs, scheduling \& caching coordination feature can enable the necessary balance between the necessary amount of acceleration and the number of UEs accessing the network.

\section{CONCLUSIONS}

Satellite network can be complementary to next generation cellular communication networks. This paper outlines integrated satellite and cellular network solutions focusing especially on satellite based backhauling solution. In addition to conceptual architecture design, we have also run experimental trials on both proposed satellite based backhaul and terrestrial cellular networks and evaluated their performances in terms of different observed KPIs. Our evaluations reveal significant insights on existence of possible trade-offs between traffic acceleration rate and number of UEs that can be scheduled inside the network. Based on the encountered challenges during experimental trials, we also proposed a novel and different architectural deployment suggestion to achieve equivalent performance with terrestrial networks.

\section{REFERENCES}

[1] 3GPP TS Group RAN, "Solutions for NR to support NTN," in 3GPP TR 38.821 V0.7.0 (2019-05) (Release 16), 2019.

[2] G. Ziaragkas et al. "SANSA-hybrid terrestrial-satellite backhaul network: scenarios, use cases, KPIs, architecture, network and physical layer techniques." International Journal of Satellite Communications and Networking, 35.5 (2017): 379-405.
[3] K. Liolis et al. "Use cases and scenarios of 5G integrated satellite-terrestrial networks for enhanced mobile broadband: The SaT5G approach." International Journal of Satellite Communications and Networking, 37.2 (2019): 91-112.

[4] Satis5, "Demonstrator for Satellite-Terrestrial Integration in the 5G Context" https://satis5.eurescom.eu/ [Online access: 10/07/2019].

[5] E. Zeydan et al. Big data caching for networking: Moving from cloud to edge. IEEE Communications Magazine, 54(9), pp.3642, 2016.

[6] E. Zeydan et al, "On the Impact of Satellite Communications over Mobile Networks: An Experimental Analysis", in IEEE Transactions on Vehicular Technology, (early access), 2019.

[7] Y. Hecht et al. "Methods and apparatus for optimizing tunneled traffic." WO No. 2015/198303 Al, filed on June 17, 2015.

[8] S. Ahluwalia et al "Acceleration of GTP traffic flows, over a satellite link, in a terrestrial wireless mobile communications system." US Patent No. US2016/0192235A1, issued on August 21, 2018.

[9] 3GPP Technical Specification Group Services and System Aspects, "IP network layer security ," in $3 G P P$ TS $33.210 \mathrm{V16.1.0}$ (2019-03) (Release 16);, 2019.

[10] A. Abdelsalam et al. "Robust security framework for DVB-RCS satellite networks (RSSN)." International Journal of Satellite Communications and Networking, 35.1 (2017): 17-43.

[11] Wireless Internet. "Major US Satellite Internet Providers.", https://goo.gl/F3bzUQ [Online access: 11/02/2019].

[12] ITU-R, "IMT Vision - Framework and overall objectives of the future development of IMT for 2020 and beyond", ITU-R Recommendation M.2083-0, September, 2015.

[13] Y. Nishida et al. SCTP-PF: a quick failover algorithm for the stream control transmission protocol. IETF RFC \# 7829, 2016.

[14] ITU-T, "Packet over Transport aspects - Synchronization, quality and the availability targets", ITU G.8275.1/Y.1369.1, 08/2017.

[15] Checkpoint Soft. Tech., Ltd. "Carrier Security Administrative Guide." R77.30 Rel. Notes, Jan. 2018, https://goo.gl/PgwuCz

Yekta Turk received his Ph.D. degree in Computer Engineering from Maltepe University, Istanbul, Turkey, in 2018. He is currently with Ericsson Research. His research interests are in the areas of mobile radio telecommunications and computer networks.

Engin Zeydan received his Ph.D. degree in Electrical Engineering from Stevens Institute of Technology, Hoboken, NJ, USA, in 2011. He is currently a Researcher at Centre Tecnologic de Telecomunicacions de Catalunya. His research interests are in the areas of telecommunications and big data. 